Pacific Journal of Mathematics

SPANNED AND AMPLE VECTOR BUNDLES WITH LOW 


\title{
SPANNED AND AMPLE VECTOR BUNDLES WITH LOW CHERN NUMBERS
}

\section{EDOARDo BALLICO}

\begin{abstract}
Here we classify paris $(V, E)$ with $V$ projective variety, $\operatorname{dim}(V)=$ $n, E$ ample and spanned rank-2 vector bundle and, if $n=2 k, c_{2}(E)^{k}=$ 1 , if $n=2 k+1, c_{1}(E) c_{2}(E)^{k}=2$. In both cases $V=P^{n}$ and $E$ is the direct sum of two line bundles of degree 1 .
\end{abstract}

Introduction. In the last few years a few papers appeared (e.g. [LP], [LS], [W1], [W2]) giving classifications, under suitable assumptions, of pairs $(V, E)$ with $V$ projective variety and $E$ an ample, spanned vector bundle with low Chern classes. It is natural to arise the following conjecture (which is proved in 2.2 in a stronger form if the bundle is the direct sum of $r$ line bundles):

Conjecture. Fix integers $n, r s, i_{1}, \ldots, i_{s}$, with $s>0,0<r, 0<$ $i_{t} \leq \min (r, n), i_{1}+\cdots+i_{s}=n$. Fix an irreducible, complete variety $V, \operatorname{dim}(V)=n$, and an ample vector bundle $E, E$ spanned by global sections. Then

$$
c_{i-1}(E) \cdots c_{i_{s}}(E) \geq\left(\begin{array}{c}
r \\
i_{1}
\end{array}\right) \cdots\left(\begin{array}{c}
r \\
i_{s}
\end{array}\right)
$$

and if we have equality, then $V \cong \mathbf{P}^{n}$ and $E \cong r \mathscr{O}_{V}(1)$.

Here we work over an algebraically closed field $\mathbf{K}$ and prove the following results.

THEOREM 1. Fix an even integer $n=2 k>0$. Let $V$ be an integral complete variety and $E$ a rank-2 ample vector bundle on $V, E$ spanned by its global sections and with $c_{2}(E)^{k}=1$. Assume either $V$ CohenMacaulay or char $(\mathbf{K})=0$. Then $V \cong \mathbf{P}^{n}$ and $E \cong 2 \mathscr{O}_{V}(1)$.

THEOREM 2. Fix an odd integer $n=2 k+1>0$. Let $V$ be an integral complete variety and $E$ a rank-2 ample vector bundle on $V, E$ spanned by its global sections and with $c_{1}(E) c_{2}(E)^{k}=2$. Assume either $V$ Cohen-Macaulay or $\operatorname{char}(\mathbf{K})=0$. Then $V \cong \mathbf{P}^{n}$ and $E \cong 2 \mathscr{O}_{V}(1)$. 
For a fixed variety $V$, Theorem 1 follows from the conjecture of [LS]; hence Theorem 1 was known in several cases proved in [LP], [W1], 3.4, [W2].

This paper is dedicated to Alessandra.

Notations. For a projective space $X$, we write $\mathscr{O}(1)$ instead of $\mathscr{O}_{X}(1)$ when there is no danger of misunderstanding. A vector bundle is called spanned if it is spanned by its global sections. We use $|L|$, $L \in \operatorname{Pic}(Y)$, for the linear system associated to the sections of $L$.

\section{Proof of Theorem 1.}

LEMMA 1.1. Let $V$ be an integral complete variety, $\operatorname{dim}(V)=n$, and $E$ an ample vector bundle on $V, \operatorname{rk}(E)=r, E$ spanned by a linear subspace $W$ of its sections. Then $\operatorname{dim}(W) \geq n+r$.

Proof. Set $L:=\mathscr{O}_{P(E)}(1)$. Using $(\mathbf{P}(E), L)$ instead of $(V, E)$ we reduce to the case $r=1$. Now use that the map induced by $|L|$ is finite by the ampleness of $E$.

We omit the proof of the following general result suggested by the referee, since it will be either used in cases (e.g. under assumption (\$) in the proofs of Theorems 1 and 2) in which the existence of a suitable section with zero-locus of the right codimension is trivial or proved directly (claim in the proof of 1.3).

LEMMA 1.2. Let $E$ be a rank- $v$ ample vector bundle on an $n$-fold $X$. Assume that $E$ is spanned by its sections. Let $x_{1}, \ldots, x_{k}$ be $k$ points in $X$. If $k v \leq \operatorname{dim}(X)$, then there is $s \in H^{0}(E)$ such that $\left\{x_{1}, \ldots, x_{k}\right\} \subseteq(s)_{0}$ and $\operatorname{codim}(s)_{0}=v$. Furthermore we may assume that $x_{2}$ is a tangent vector at $x_{1}$.

LEMMA 1.3. Let $S$ be an integral complete surface and $E$ a rank-2 spanned ample vector bundle with $c_{2}(E)=1$. Then $(S, E) \cong\left(\mathbf{P}^{2}, 2 \mathscr{O}(1)\right)$.

Proof. If $S$ is normal, the result is well known (see e.g. [B] if $\operatorname{char}(\mathbf{K})>0)$. Assume that $S$ is not normal. Let $p: S^{\prime} \rightarrow S$ be the normalization. Let $E^{\prime}:=p^{*}(E)$. We have $\left(S^{\prime}, E^{\prime}\right) \cong\left(\mathbf{P}^{2}, \mathscr{O}(1)\right)$. Fix a nonnormal point $x \in S$, hence with length $\left(p^{-1}(x)\right)>1$.

Claim. There is a section $s$ of $E$ with $x \in(s)_{0}$ and $\operatorname{codim}\left((s)_{0}\right)=2$.

Assume the claim. Then $p^{*}(s)$ is a section of $E^{\prime}$ vanishing in codimension 2. Since $c_{2}\left(E^{\prime}\right)<$ length $\left(p^{-1}(x)\right)$, we get a contradiction. 
Proof of the claim. Let $F$ be the fiber of the projection $t: \mathbf{P}(E) \rightarrow S$ over $x$ and $L:=\mathscr{O}_{\mathbf{P}(E)}(1)$ the tautological line bundle. Let $h: \mathbf{P}(E) \rightarrow$ $|L|$ be the map induced by $|L|$. Since $L$ is spanned, $h(F)$ is a line. Since $L$ is ample, $h^{-1}(h(F))$ is a curve. Set $A:=t\left(h^{-1}(h(F))\right.$, and let $A(1), \ldots, A(s)$ be the irreducible components of $A$ of dimension 1 (if any). Let $P(i)$ be a general point of $A(i)$. Since $h^{-1}(h(F)) \cap t^{-1}(P(i))$ is finite, a general section of $E$ vanishing at $x$ does not vanish at $P(i)$. By Bertini's theorem ([K]) applied to $S \backslash A$, we get that a general section $s$ of $E$ with $x \in(s)_{0}$ vanishes only in codimension 2 .

The proof of Theorem 1 will be divided into several steps $((a), \ldots$, $(\mathrm{m}))$. It will give also Theorem 2 and most of the results stated in the next section.

Proof of Theorem 1. (a) Take $s \in H^{0}(E)$ with $X:=(s)_{0}$ of codimension 2. We want to prove that $(X, E \mid X) \cong\left(\mathbf{P}^{n-2}, 2 \mathscr{O}(1)\right)$. Since $c_{2}(E \mid X)^{k-1}=1, X$ is generically reduced and $Y:=X_{\text {red }}$ is irreducible. By the inductive assumption, $(Y, E \mid Y) \cong\left(\mathbf{P}^{n-2}, 2 \mathscr{O}(1)\right)$. If $V$ is CohenMacaulay, then $X$ is Cohen-Macaulay; since $X$ is generically reduced, it is reduced. Now assume $\operatorname{char}(\mathbf{K})=0$. Then a general section $s^{\prime}$ of $E$ has $W:=\left(s^{\prime}\right)_{0}$ reduced, hence $(W, E \mid W) \cong\left(\mathbf{P}^{n-2}, 2 \mathscr{O}(1)\right)$. Set $H:=\operatorname{det}(E)$. For every closed subscheme $Z$ of $V$, let $p_{Z}$ be the Hilbert polynomial of $\mathscr{O}_{Z}$ with respect to $H$. Using either flatness or the short exact sequences determined by $s$ and $s^{\prime}$, we see that $p_{X}=p_{W}$. Assume that $X$ is not reduced and let $k \geq 0$ be the dimension of the support of the nilradical of $\mathscr{O}_{X}$. For a fixed $Z$ and a general $D \in|L|$, we have $p_{Z \cap D}(n)=p_{Z}(n)-p_{Z}(n-1)$. Hence $p_{X \cap D}=p_{W \cap D}$. Taking $k$ general divisors of $L$, we get a contradiction.

(b) Set $T:=\left\{(s)_{0}: s \in H^{0}(E), \operatorname{codim}\left((s)_{0}\right)=2\right\}$. By the proof of the claim in the proof of 1.3 (or by 1.2 ), for every $x \in V$, there is $S \in T$ with $x \in S$.

(c) Now we prove that $V$ is smooth. Indeed by (b) and (a) for every $x \in V$ there is a smooth subvariety $S$ of codimension 2 and locally complete intersection in $V$, with $x \in S$.

(d) Now we give a few definitions. A curve $C \subset V$ is called a line if $C \cong \mathbf{P}^{1}$ and $E \mid C \cong 2 \mathscr{O}$ (1). A line $C$ is called of type $T$ if it is contained in some $S \in T$. Fix any $S \in T$. For any smooth codimension 2 subvariety $Y$ of $V$ which is an embedded deformation of $S$, we have $(Y, E \mid Y) \cong\left(\mathbf{P}^{n-2}, 2 \mathscr{O}(1)\right)$ by the invariance of Chern numbers under deformations. Call $G$ the set of such $Y$. A line is called of type $G$ if it is contained in some $Y \in G$. 
(e) Now we prove that for all $x, y \in V, x \neq y$, there is $S \in T$ with $\{x, y\} \subset S$.

Proof. For any $z \in V$, let $F(z)$ be the fiber over $z$ of the projection $\mathbf{P}(E) \rightarrow V$. Let $L:=\mathscr{O}_{\mathbf{P}(E)}(1)$ be the tautological line bundle and $h: \mathbf{P}(E) \rightarrow|L|$ be the induced map. Since $L$ is spanned, $h(F(z))$ is a line for all $z$, hence $h(F(x)) \cup h(F(y))$ spans a linear space $O$ of dimension at most 3 . We conclude as in the proof of the claim in the proof of 1.3.

(f) By (e) for all $x, y \in V$ with $x \neq y$ there is a line of type $T$ containing $x$ and $y$. Now we want to prove that there is a unique line containing $x$ and $y$. Take $S \in T$ with $\{x, y\} \subset S$ and let $C$ be a line containing $x$ and $y$. Since $E \mid C \cong 2 \mathscr{O}(1)$, any section of $E$ vanishing on $x$ and $y$ vanishes on $C$. Thus $C \subset S$, hence the uniqueness of the line containing $x$ and $y$. Hence every line is of type $T$. Since $E \mid S$ is the normal bundle to $S$ in $V$, every line is a smooth point of the Hilbert scheme of $V$. Write $(x, y)$ for the line containing $x$ and $y$, $x \neq y$.

(g) Now assume the existence of a divisor $D>0$ with $h^{0}(E(-D)) \neq$ 0 . Fix $x, y, z$ in $V$ with $x \in D, y \notin D$. Take $X \in T, X$ containing $x$, uy. Since $D \cap S$ is a positive divisor, we have $\mathscr{O}(D) \mid S \cong \mathscr{O}_{S}(1)$. Fix a section $s$ of $E(-D), s \neq 0$. We have seen that $s$ does not vanish on $S$ or vanishes identically on $S$. By (e) we get easily that $s$ vanishes nowhere on $S$, hence it does not vanish at $x$ or at $y$. Thus $s$ does not vanish at all and shows that $E(-D)$ is the extension of a line bundle $M$ by the trivial line bundle. Set $R:=M(D)$. We have seen that $R \mid S \cong \mathscr{O}_{S}(1)$ for every $S \in T$. Since $R$ is a quotient of $E, R$ is ample and spanned. Fix $A \in|R|, u \in A, z \in A, v \notin A, S \in T, Q \in T$ with $u \in S \cap Q$, $v \in S, z \in Q$. Since $R \mid S \cong \mathscr{O}_{S}(1)$, one sees that $A$ is smooth and irreducible, and that for any two points of $A$, the line containing them is contained in $A$. Fix $W \in T$. Since $h^{0}(R \mid W)=n-1 \leq h^{0}(R)-2$, there are $A, B \in R$ with $A \neq B, W \subseteq(A \cap B)$. Since $A \cap B$ contains the line joining any two of its points, $(A \cap B)_{\text {red }}=W$. Fix $a \in W$ and $C \in|R|$ with $a \notin C$. Since $V$ is the union of the lines $(a, t), t \in C$, there is a line $(a, m)$ not tangent to $A$ at $a$. Take $B^{\prime}$ in the pencil of $R$ spanned by $A$ and $B$, with $m \in B^{\prime}$. Then $A \cap B^{\prime}$ is smooth at $a$, hence everywhere i.e. $(A \cap B)=W$. Thus $R^{n}=1$. Since $R$ is ample and spanned, and $V$ is smooth $V \cong \mathbf{P}^{n}, R \cong \mathscr{O}(1)$. By (e) for every line $I$ of $V, E \mid I \cong 2 \mathscr{O}_{I}(1)$, hence $E \cong 2 \mathscr{O}(1)$ (e.g. use [E]). 
(h) From now on in this section, we make the following assumption $(\$)$ :

$$
\text { For divisors } D>0, h^{0}(E(-D))=0 \text {. }
$$

By (g) to prove Theorem 1 it is sufficient to assume (\$) and find a contradiction.

(i) First assume $h^{0}(E)>6$; by 1.1 this is satisfied if $k>2$. Fix any 3 points $x, y, z$ of $V$. By assumption there is $s \in H^{0}(E)$ with $s(x)=x(y)=s(z)=0, s \neq 0$. By $(\$)$ there is $S \in T$ with $\{x, y, z\} \subset S$. We want to check that if $h^{0}(E)=6$ there is $S \in G$ with $\{x, y, z\} \subset S$ and that if $z \notin(x, y)$, such a surface $S$ is unique. First the uniqueness. If $S, S^{\prime}$ are surfaces with this property, $S \cap S^{\prime}$ contains the line joining any two of its points, hence $S=S^{\prime}$. Counting dimensions, we see that for general $x, y, z$ there is $S \in G$ containing them. Since by the proof of (a) $G$ is a complete family, this is true for all $x, y, z$; alternatively one can use the union of the lines $(z, t)$ with $t \in(x, y)$.

(j) For every $S \in G$ and every $P \notin S$, let $D(P, S)$ be the union of the lines $(P, t)$ with $t \in S . D(P, S)$ is a divisor. First we check that for any $x, y \in D(P, S),(x, y) \subset D(P, S)$ (hence in particular $D(P, S)$ is irreducible). Take $u, v \in S$ such that $x \in(u, P), y \in(v, P)$. Fix $t \in(x, y)$. By (i) there is $W \in G$ with $\{u, v, P\} \subset W$, hence with $t \in W$ and with $(t, P) \in W$; hence $(t, P) \cap(u, v) \neq \varnothing$, i.e. $t \in D(P, S)$. Note that the divisors $D(P, S)$ and $D\left(P^{\prime}, S^{\prime}\right)$ are algebraically equivalent. Hence for any two points $a, b \in V$, there is a divisor $D$ algebraically equivalent to $D(P, S)$ and with $a \in D, b \notin D$; by Nakai's ampleness criterion $D(P, S)$ is ample.

(k) By $(\mathrm{j})$ any line of type $T$ not contained in $D(P, S)$ intersects $D(P, S)$ at most at a point. Fix a point $x$ of $D(P, S)$. By $(\mathrm{k})$ there is a line $F$ of type $T$ intersecting $D(P, S)$ only at $x$ and transversally. Thus $\mathscr{O}(D(P, S)) \mid F \cong \mathscr{O}(1)$. Thus the same is true for all lines (by (f) they are of type $T)$, hence $\mathscr{O}(D(P, S)) \mid S=\mathscr{O}(1)$. Fix $y \in D(P, S)$ and any line of type $T$ through $y$ and not contained in $D(P, S)$; since $D(P, S) \neq V$, the existence of such a line follows from (i) and (j); we get that $D(P, S)$ is smooth at $y$ for every $y$. Since for suitable $P^{\prime}$, we have $D(P, S) \cap D\left(P^{\prime}, S\right)=S$ (set-theoretically), $S$ is ample in $D(P, S)$ by the last part of (i).

(1) Set $A:=D(P, S)$.

Claim. $E \mid A \cong 2 \mathscr{O}_{A}(A)$. 
Proof of the claim. Let $\mathscr{I}$ be the ideal sheaf of $S$ in $A$; let $S(k)$ be the $k$ th infinitesimal neighborhood of $S$ in $A$, with ideal sheaf $\mathscr{I}^{k+1}$. Set $F:=\operatorname{Hom}\left(2 \mathscr{A}_{A}(A), E \mid A\right)$. Since $E$ is ample in $A$, there is an integer $k>0$ such that $h^{1}\left(A, F \otimes \mathscr{I}^{k+1}\right)=0$, thus $H^{0}(A, F) \rightarrow$ $H^{0}(S(k), F \mid S(k))$ is surjective. Since $\mathscr{I}^{k} / \mathscr{J}^{k+1} \cong \mathscr{O}_{S}(k)$, from the isomorphism $E \mid S \cong 2 \mathscr{O}_{S}(1)$ and the exact sequence

$$
0 \rightarrow \mathscr{I}^{t} / \mathscr{I}^{t+1} \otimes F \rightarrow F|S(t) \rightarrow F| S(t-1) \rightarrow 0
$$

we find that the restriction map $H^{0}(S(k), F \mid S(k)) \rightarrow H^{0}(S, F \mid S)$ is surjective. Thus there is $c \in H^{0}(A, F)$ which induces the isomorphism between $2 \mathscr{O}_{S}(1)$ and $E \mid S$. Since $S$ is ample in $A$, every divisor of $A$ intersects $S$. Thus $c$ induces an isomorphism at every point of $A$ (take the determinant!).

(m) The same proofs as in (l) give that $E=2 \mathscr{O}(A)$, containing (\$). The proof of Theorem 1 is over.

\section{Proof of Theorem 2.}

Proof of Theorem 2. First assume $n=1$. Let $h$ be the morphism from $V$ to a suitable Grassmannian Grass induced by $H^{0}(E)$. By assumption (for the Plucker embedding) $\operatorname{deg}(h) \operatorname{deg}(h(V))=2$. Thus $h(V)$ is smooth and rational. If $\operatorname{deg}(h)=2, h(V)$ is a line and the restriction of the universal quotient bundle of Grass to $h(V)$ is not ample (see e.g. [P], p. 123), contradicting the ampleness of $E$. Thus $h$ is an isomorphism and $E$ must be the direct sum of two line bundles of degree 1. If $n>3$, the inductive proof of Theorem 1 works. If $n=3$, however that proof has to be modified (in particular point (e) and its consequences). Thus we assume $n=3$ and use the terminology "line of type $T$ or of type $G$ " as in the previous section.

(1) As in (b) of $\S 1$, for every $P \in W$, there is a line $C$ of type $T$ with $P \in C$. As in (c) of $\S 1$ this implies the smoothness of $V$.

(2) First assume the existence of a divisor $D>0$ with $h^{0}(E(-D)) \neq$ 0 . By (1) there is a line $A$ of type $T$ not contained in $D$. Since $E \mid D$ is ample, $c_{2}(E \mid D) \neq 0$, hence for every line $C$ of type $T, C \cap D \neq \varnothing$. Thus $\mathscr{O}(D) \mid C$ has degree 1 for every $C$ of type $T$. Fix $s \in H^{0}(E)$ with $(s)_{0}=A$ and $t \in H^{0}(E(-D)), t \neq 0$. Then $s \mid D$ shows that $c_{2}(E \mid D)=1$. By $1.3 D \cong \mathbf{P}^{2}, E \mid D \cong 2 \mathscr{O}(1)$. Note that $t$ either vanishes identically on a line not in $D$ or has no zero there. Fix a point $P \in V$. By 1.1 and Bertini's theorem $([\mathbf{K}])$, we see that there are infinitely many lines of type $T$ through $P$. Thus we see that if $t$ vanishes at $P$, it vanishes in codimension 1. Enlarging if necessary $D$, we get a contradiction. 
Thus $t\left(\mathscr{O}_{V}\right)$ is a subline bundle of $E(-D)$; let $M:=E(-D) / s\left(\mathscr{O}_{V}\right)$, $R:=M(D)$, hence $R$ ample and spanned. Fix $A \in|R|$. As before we see that $(A, E \mid A) \cong\left(\mathbf{P}^{2}, 2 \mathscr{O}(1)\right)$. Since $h^{0}(V, A)>3, A$ contains a line $B$ of type $T$. Thus $\mathscr{O}(A) \mid A \cong \mathscr{O}(1)$, hence $A^{3}=1$, and we get the thesis.

(2) From now on, we assume the following assertion (\$):

$$
\text { there is no divisor } D>0 \text { with } h^{0}(E(-D)) \neq 0 \text {. }
$$

By 1.1 for any length 2 subscheme $X$ of $V$ there is a non-zero section of $E$ vanishing there. By $(\$)$ there is a line of type $T$ containing $X$. Such a line is unique by $(\$)$ (even taking lines not of type $T$ ). The uniqueness implies that every line is of type $T$.

(3) Fix any line $S$ and $P \notin S$. Let $A=D(P, S)$ be the union of the lines $(P, t)$ with $t \in S$. Let $Q$ be the image of $H^{0}(E)$ into $H^{0}(A, E \mid A)$ by the restriction map. Take a general $s \in Q$ with $s(P)=0$. By Bertini's theorem we see that $s$ vanishes only in codimension 2 on $A$. By the last part of (2) we see that $P=(s)_{0}$ as a scheme. Thus by 1.3 $(A, E \mid A) \cong\left(\mathbf{P}^{2}, 2 \mathscr{O}(1)\right)$. In particular every section of $E \mid A$ vanishing on a scheme of length 2 vanishes on a "line" of $A$. Thus by the last statement in (2) every line intersecting $A$ at more than one point is contained in $A$. Taking $D\left(P^{\prime}, S\right)$ for general $P^{\prime}$, we get $A^{3}=1$, hence $V=\mathbf{P}^{3}$. By [E] $E$ splits and Theorem 2 is proved.

REMARK 2.1. Fix $(V, E)$. A line in $V$ is a smooth rational curve $C$ such that $E \mid C$ is a direct sum of line bundles of degree 1 . Here are some properties a pair $(V, E)$ can have: (i) through a general point there is a line; (ii) for two general points there is a line; (iii) for every pair of points there is a line containing them. In (ii) and (iii) we can ask also the uniqueness of the line. The proofs of Theorems 1 and 2 , show that (ii) is true if in the statement of the theorems we omit the Cohen-Macaulay assumption; furthermore no pair $(V, E)$ exists if in the statement of Theorem 2 we take $c_{1}(E) c_{2}(E)^{k}=1$. One gets similar results, for instance if $r=3, n=1+3 k, c_{1}(E) c_{3}(E)^{k}=3$ (no such pair exists if $\left.c_{1}(E) c_{3}(E)^{k}<3\right)$ and in a few similar cases.

Now we show that the conjecture holds (in a stronger form) for vector bundles which are direct sum of ample, spanned line bundles.

Proposition 2.2. Fix integers $r, n, s, i_{1}, \ldots, i_{s}$ with $r>0, n>0$, $s>0,0<i_{t} \leq \min (r, n)$ for all $t, i_{1}+\cdots+i_{s}=n$. Let $V$ be a complete, integral variety and $L_{1}, \ldots, L_{r}$ be ample and spanned line bundles on 
$V$. Set $E:=L_{1} \oplus \cdots \oplus L_{r}$ and $c=c_{i_{1}}(E) c_{i_{2}}(E) \cdots c_{i_{s}}(E)$. Then

$$
c \geq d:=\left(\begin{array}{c}
r \\
i_{1}
\end{array}\right) \cdots\left(\begin{array}{c}
r \\
i_{s}
\end{array}\right)
$$

and if $c=d$, then $V \cong \mathbf{P}^{n}$ and $L_{t}$ has degree one for all $t$.

Proof. The intersection number of any $n$ ample line bundles is $>0$. The result follows immediately from the following claim.

Claim. Fix any $n$ ample, spanned, line bundles $M_{1}, \ldots, M_{n}$ in $V$. If their intersection number is one, then $V \cong \mathbf{P}^{n}$ and each $M_{t}$ has degree one.

Proof of the claim. By induction on $n$, the cases with $n=1$ and $n=2$ being left to the reader; for $n=2$ use for instance Hodge index theorem. Assume $n \geq 3$. Take $A \in\left|M_{1}\right|$. By induction we get $A \cong \mathbf{P}^{n-1}$ and each $M_{t} \mid A, t>1$, has degree one. Set $U:=M_{1}$, $J:=M_{2}$. We get $U J^{n-1}=1$. Taking $B \in|J|$, we get $B \cong \mathbf{P}^{n-1}$ and $J B$ of degree one. Thus $J^{n}=1$, and the claim is easy.

\section{REFERENCES}

[B] E. Ballico, On globally generated vector bundles on surfaces with $c_{2}=1$, Geometriae Dedicata, 27 (1988), 319-324.

[E] L. Ein, Stable vector bundles on projective spaces in char $p>0$, Math. Ann., 254 (1980), 53-72.

[K] S. L. Kleiman, The transversality of a general translate, Compositio Math., 28 (1974), 287-297.

[LP] A. Lanteri and M. Palleschi, Some characterizations of projective bundles and projective spaces, Geometriae Dedicata, 14 (1983), 203-208.

[LS] A. Lanteri and A. J. Sommese, $A$ vector bundle characterization of $\mathbf{P}^{n}$, preprint.

[P] A. Papantonopoulou, Curves in Grassmann varieties, Nagoya Math. J., 66 (1977), 121-136.

[W1] J. Wisniewski, Length of extremal rays and applications, Ph. D. Thesis, Notre Dame Univ. 1987.

[W2] _ Length of extremal rays and a characterization of projective space, preprint.

Received February 2, 1988 and in revised form, June 14, 1988.

UNIVERSITY OF TRENTO

38050 Povo (TN)-ITALY 


\section{PACIFIC JOURNAL OF MATHEMATICS EDITORS}

\author{
V. S. VARAdarajan \\ (Managing Editor) \\ University of California \\ Los Angeles, CA 90024-1555-05 \\ Herbert Clemens \\ University of Utah \\ Salt Lake City, UT 84112 \\ ThOMAS ENRIGHT \\ University of California, San Diego \\ La Jolla, CA 92093
}

R. FINN

Stanford University

Stanford, CA 94305

HeRmann FlaschKa

University of Arizona

Tucson, AZ 85721

VAUGHAN F. R. Jones

University of California

Berkeley, CA 94720

SteVen KercKhofF

Stanford University

Stanford, CA 94305

\section{ROBION KIRBY}

University of California

Berkeley, CA 94720

C. C. MOORE

University of California

Berkeley, CA 94720

HAROLD STARK

University of California, San Diego

La Jolla, CA 92093

\section{ASSOCIATE EDITORS}
R. Arens
E. F. BeCKenbaCH
B. H. NEUMANN
F. Wolf
K. YoshidA (1906-1982)

\section{SUPPORTING INSTITUTIONS}

UNIVERSITY OF ARIZONA

UNIVERSITY OF BRITISH COLUMBIA

CALIFORNIA INSTITUTE OF TECHNOLOGY

UNIVERSITY OF CALIFORNIA

MONTANA STATE UNIVERSITY

UNIVERSITY OF NEVADA, RENO

NEW MEXICO STATE UNIVERSITY OREGON STATE UNIVERSITY

\author{
UNIVERSITY OF OREGON \\ UNIVERSITY OF SOUTHERN CALIFORNIA \\ STANFORD UNIVERSITY \\ UNIVERSITY OF HAWAII \\ UNIVERSITY OF TOKYO \\ UNIVERSITY OF UTAH \\ WASHINGTON STATE UNIVERSITY \\ UNIVERSITY OF WASHINGTON
}

The Supporting Institutions listed above contribute to the cost of publication of this Journal, but they are not owners or publishers and have no responsibility for its content or policies.

Mathematical papers intended for publication in the Pacific Journal of Mathematics should be in typed form or offset-reproduced (not dittoed), double spaced with large margins. Please do not use built up fractions in the text of the manuscript. However, you may use them in the displayed equations. Underline Greek letters in red, German in green, and script in blue. The first paragraph must be capable of being used separately as a synopsis of the entire paper. In particular it should contain no bibliographic references. Please propose a heading for the odd numbered pages of less than 35 characters. Manuscripts, in triplicate, may be sent to any one of the editors. Please classify according to the scheme of Math. Reviews, Index to Vol. 39. Supply name and address of author to whom proofs should be sent. All other communications should be addressed to the managing editor, or Elaine Barth, University of California, Los Angeles, California 90024-1555-05.

There are page-charges associated with articles appearing in the Pacific Journal of Mathematics. These charges are expected to be paid by the author's University, Government Agency or Company. If the author or authors do not have access to such Institutional support these charges are waived. Single authors will receive 50 free reprints; joint authors will receive a total of 100 free reprints. Additional copies may be obtained at cost in multiples of 50 .

The Pacific Journal of Mathematics is issued monthly as of January 1966. Regular subscription rate: $\$ 190.00$ a year (5 Vols., 10 issues). Special rate: $\$ 95.00$ a year to individual members of supporting institutions.

Subscriptions, orders for numbers issued in the last three calendar years, and changes of address should be sent to Pacific Journal of Mathematics, P.O. Box 969, Carmel Valley, CA 93924, U.S.A. Old back numbers obtainable from Kraus Periodicals Co., Route 100, Millwood, NY 10546.

The Pacific Journal of Mathematics at P.O. Box 969, Carmel Valley, CA 93924 (ISSN 0030-8730) publishes 5 volumes per year. Application to mail at Second-class postage rates is pending at Carmel Valley, California, and additional mailing offices. Postmaster: send address changes to Pacific Journal of Mathematics, P.O. Box 969, Carmel Valley, CA 93924.

PUBLISHED BY PACIFIC JOURNAL OF MATHEMATICS, A NON-PROFIT CORPORATION Copyright (C) 1989 by Pacific Journal of Mathematics 


\section{Pacific Journal of Mathematics \\ Vol. 140, No. $2 \quad$ October, 1989}

Edoardo Ballico, Spanned and ample vector bundles with low Chern numbers

Marcy Mason Barge, Richard Swanson and Russell Bruce Walker,

Conjugacy class structure of smooth hyperbolic sectors . ........... 217

Jeffrey Stephen Fox, Adeles and the spectrum of compact nilmanifolds . . ..233

Robert D. Little, Homotopy complex projective spaces with divisible

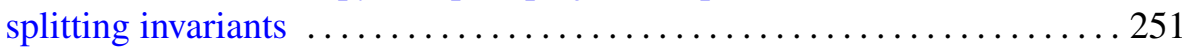

M. Scott Osborne and Garth William Warner, Jr., The Selberg trace formula. VII. Application of the truncation process to the continuous

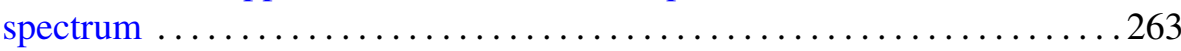

John R. Stembridge, On the eigenvalues of representations of reflection

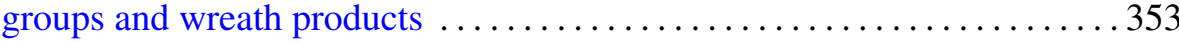

Ibrahim Salama, Corrections to: "Topological entropy and recurrence of

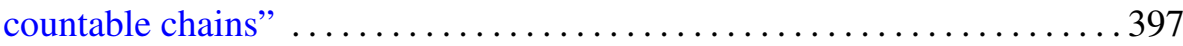

Robert Greene and Hung-Hsi Wu, Addendum to: "Lipschitz convergence of Riemannian manifolds" ............................... 398

Kayoko Shikishima-Tsuji, Correction to: "Galois theory of differential fields of positive characteristic" 